\title{
Association between screening family medical history in general medical care and lower burden of cancer worry among women with a close family history of breast cancer
}

Robert Gramling, $M D^{1}$, David Anthony, $M D^{1}$, Jan Lowery, $P h D^{2}$, Lori Ballinger, $M S^{3}$, Deb $M a^{4}$, Rana Habbal, $M A^{5}$, Nancy Leighton, $R D^{5}$, Deborah Bowen, $P h D^{6}$

\begin{abstract}
Purpose: Soliciting family medical history (FMH) is the initial step in the process of screening for heritable cancer risk in medical care. We investigate whether recent solicitation of FMH in general medical care is associated with cancer worry among a sample of women having a first-degree relative with a breast cancer diagnosis. Methods: Surveys were mailed to women registered with the Cancer Genetics Network having a first-degree relative with a breast cancer diagnosis and a regular source of medical care. The independent measure consisted of two items for solicitation of FMH based on validated measures of clinical interactions with one's physician; the dependent measure was a novel measure of cancer worry based on validated patient-centered measure of distress; and the secondary measures were 6-point scales for perceived likelihood of developing breast cancer and perceived severity of breast cancer as a health outcome. Results: A total of 353 women responded and met eligibility criteria (76.4\% minimum response rate). One fifth reported no cancer worry during the past 4 weeks. After adjustment for age, education, pedigree features, and clustering within families, recent FMH solicitation was associated with lower odds of cancer worry (odds ratio $=0.58$; 95\% confidence interval $=0.51-0.70)$. FMH solicitation was associated with lower perceptions of the severity of developing breast cancer but not with the perception of cancer likelihood. Conclusions: Our data do not support the hypothesis that FMH solicitation in general medical practice causes cancer worry. In fact, we observed a protective association possibly explained by influences on perceptions of breast cancer severity. Prospective research among less select populations is necessary. Genet Med 2005:7(9):640-645.
\end{abstract}

Key Words: screening, genetics, primary care, psychosocial functioning, cancer worry

Soliciting a family medical history $(\mathrm{FMH})$ is the first step toward identifying women with clinically relevant degrees of genetic breast cancer risk. For this purpose, FMH constitutes a genetic screening tool ${ }^{1-5}$ that is commonly used in medical practice $^{4,6}$ and is endorsed by the U.S. Surgeon General. ${ }^{7}$ Evidence is mounting in support of tailoring preventive care for women with high degrees of genetic breast cancer risk, ${ }^{8-10}$ yet little evidence is available to describe the psychosocial impact of FMH screening processes designed to identify such women.

From the ${ }^{I}$ Brown Medical School, Providence, Rhode Island; ${ }^{2}$ University of Colorado Health Sciences Center, Denver, Colorado; ${ }^{3}$ University of New Mexico Health Sciences Center, Albuquerque, New Mexico; ${ }^{4}$ Huntsman Cancer Institute, University of Utah, Salt Lake City, Utah; ${ }^{5}$ University of California, Irvine, California; ${ }^{6}$ Fred Hutchinson Cancer Research Center, Seattle, Washington.

Robert Gramling, MD, Center for Primary Care and Prevention, Memorial Hospital of Rhode Island, 111 Brewster Street, Pawtucket, RI 02860.

Received: June 14, 2005.

Accepted: August 9, 2005

DOI: 10.1097/01.gim.0000187123.76699.e9
As computer-based supports for pedigree analysis emerge, ${ }^{11-15}$ understanding the beneficial and adverse effects of screening FMH becomes a salient and pressing public health concern.

Anxiety is a frequent unintended outcome of many screening interventions. ${ }^{16}$ Worry about developing breast cancer is a specific symptom of anxiety that can promote dysfunctional coping behaviors ${ }^{17}$ and is more indicative of which women will seek DNA testing than general anxiety. ${ }^{18}$ Although it has been established that many women with a close FMH of breast cancer experience cancer worry ${ }^{19-23}$ and that such worry is greater among those women having a family history than those without, ${ }^{22}$ it remains unclear how handling of FMH information by one's regular doctor impacts preexisting cancer worry. Understanding whether, and how, current FMH screening practices impact cancer worry is essential to guide subsequent translation of genetic advances into clinical practice settings.

This study was designed to describe the relationships between recent exposure to FMH solicitation in the context of routine medical care and the presence of worry about developing breast cancer. 


\section{MATERIALS AND METHODS}

\section{Design}

We performed a cross-sectional survey using a mailed questionnaire during the winter of 2003 to the spring of 2004 among women registered with the Cancer Genetics Network (CGN).

\section{Setting}

The CGN ${ }^{24-26}$ is a federally funded, collaborative project that aims to provide infrastructure for studying the genetic basis of cancer susceptibility; to understand the psychosocial, ethical, legal, and public health issues related to genetic susceptibility to cancer; and to investigate the best means for incorporating such information into medical care. One major objective of the CGN is to recruit individuals having a family history, but no personal history, of cancer to create a contact registry for future studies related to cancer susceptibility. All consenting participants complete a baseline telephone interview consisting of a four-generation pedigree and sociodemographic information. All enrollees agree to be approached for participation in future research studies and are contacted annually to update their baseline data.

The CGN comprises eight recruitment sites across the United States. Half of these sites recruit participants with population-based methods, and the other half use clinic-based recruitment strategies. ${ }^{26}$ This study was restricted to participants recruited to the CGN by population-based strategies to minimize the selection bias represented by recruiting individuals from high-risk cancer centers and genetic counseling programs. All CGN recruitment, interviews, and follow-up protocols were standardized across centers and approved by the site institutional review board for protection of human subjects.

\section{Participants}

We mailed a cover letter, refusal card, and survey to 585 women registered with the CGN who met the following criteria as established by the baseline telephone interview completed on recruitment to the CGN: 25 to 65 years of age, family history of breast cancer in a first-degree relative (FDR), and no personal history of a major cancer (i.e., any cancer other than nonmelanomatous skin cancer). Final eligibility for this study included women who had a regular source of medical care and who visited their doctors within the past year. These final criteria could be assessed only for those completing the study questionnaire. Our response rate calculations assume that all nonresponders would have met these final eligibility criteria and therefore are likely to have biased our response rates downward.

\section{Data collection}

The 7-page questionnaire consisted of 34 multiple-choice items. Participants were provided postage-paid return envelopes and refusal postcards. Ten days after the initial mailing, three attempts were made to contact nonresponders by telephone over a 1-week period. Those contacted could refuse par- ticipation or request another questionnaire. Those unable to be reached by telephone were mailed a second questionnaire within 3 weeks after the initial mailing.

\section{Measures}

The independent and dependent measures were designed explicitly for the goals of this study and underwent qualitative evaluation before arrival at the final items. All measures were subsequently pretested in the final instrument for question comprehension, response option comprehension, and response burden among 25 women of diverse educational backgrounds.

\section{Independent variable}

\section{Family medical history solicitation}

We defined FMH solicitation to be marked by at least the active collection of first-degree family history. FMH can be solicited by written or spoken methods in the clinical setting. Therefore, we operationalized our definition by including the following two questions: "Have you ever filled out forms for your regular doctor that asked you for information about health problems in your immediate family (parents, brothers or sisters)?" or "Has your regular doctor ever asked you for information about health problems in your immediate family (parents, brothers or sisters)?"

Response options for both of the above independent variable items included "Yes, in the last year," "Yes, more than 1 year ago," "Yes, I don't remember when," and "No." A response of "Yes, in the past year" to either item was considered positive for FMH solicitation. These items were adapted from a similar validated measure of patient interactions with their regular physician. ${ }^{27}$ To reduce recall error, we limited our timeframe to the last year. Self-report about topics arising in the medical encounter have been demonstrated to be valid, ${ }^{28}$ particularly when the issue is relevant to the patient.

\section{Main dependent variable}

\section{Cancer worry}

We considered using the Cancer Worry Scale (CWS), but chose not to do so for the following reasons: The CWS has neither thresholds nor minimally important differences established by which to interpret the clinical significance of mean scores; others have challenged the validity of the CWS as a linear scale; 22 many versions of the CWS exist in the literature;21,22,29,30 and prior related work demonstrates a floor effect with the CWS 22 in which much of the variability at the low end of the scale is captured by one item related to frequency of cancer-related thoughts, a measure of awareness rather than distress. The Impact of Events scale ${ }^{18}$ also has been used to measure breast cancer worry; however, the Impact of Events scale addresses levels of distress related to more traumatic life events than we thought FMH solicitation would represent. Therefore, we chose to develop a new patient-centered measure with explicit goals of establishing a clinically interpretable and sensitive measure of whether the individual is 
bothered by any thoughts about her breast cancer risk during the past month or not.

Given these concerns, we modeled our measure of cancer worry on the validated wording and pictograph metric (emotive faces) of the Dartmouth COOP Chart triage measure for emotional distress: ${ }^{31}$ "During the past 4 weeks. . how much have you been bothered by thoughts or worry about your chances of getting breast cancer?" Responses included "Not at all [happy face]," "Slightly," "Moderately [neutral face]," "Quite a bit," and "Extremely [sad face]."

\section{Intermediate outcomes}

We were interested in gaining insight into processes by which FMH solicitation might influence cancer worry. We considered both the perceived likelihood of developing breast cancer and the perceived severity of breast cancer as a health outcome for potential explanatory variables for this relationship because identification of an FDR during FMH screening is likely to stimulate some consideration of the probability of developing breast cancer and the survivability of breast cancer. These explanatory variables represent intermediates in the association under study, so they are considered to be neither confounders nor effect modifiers. For both constructs, we used single-item measures with 6-point scales that have been advocated in the risk perception literature. ${ }^{32,33}$ For perceived likelihood, we asked the following: "How likely do you believe it is that you will get breast cancer someday?"(Response options: 1 $=$ No chance, $6=$ Certain to happen.) For perceived severity, we asked the following: "Getting breast cancer would be a very serious problem." (Response options: 1 = Strongly disagree, 6 $=$ Strongly agree.) We assessed the association with FMH using the Student $t$ test for crude analyses and a multiple linear regression model for adjustment.

\section{Potential confounder/effect modifying variables}

We considered the following variables as potential confounders or effect modifiers of our main association between FMH solicitation and cancer worry: age, education, and presence of higher risk family history characteristics. All eligible women had at least one FDR diagnosis with breast cancer. We defined high-risk family history characteristics by the method advocated by Scheuner et al.: ${ }^{2}$ presence of an affected male FDR, multiple affected FDRs, or an FDR with age of onset of 50 years or less. We chose these criteria because they are all manifest in a first-degree FMH and are likely to attract physician attention on solicitation of FMH. We categorized women with no such characteristics $(\mathrm{N}=173)$ into a lower family history risk group and those with at least one of the above criteria into a higher family history risk group $(\mathrm{N}=180)$ for the purposes of stratified analyses.

\section{Human subjects}

The development and pretesting of the measures were approved by the Miriam Hospital Review Board for Protection of Human Subjects (Rhode Island).
The main study was approved by the institutional review boards for Miriam Hospital (Rhode Island), the University of New Mexico, the University of California at Irvine, the University of Colorado Health Sciences Center, and the University of Utah.

\section{Analyses}

\section{Crude and stratified comparisons}

Odds ratios (ORs) and 95\% confidence intervals (CIs) were calculated for comparisons involving dichotomous measures. Crude and stratified analyses were conducted for the association between FMH solicitation and cancer worry to investigate potential confounding or effect modification. Standard chisquare tests were used to identify statistically different proportions.

\section{Control of confounding}

We chose to fit a multiple logistic model for all potential confounding variables and proceeded with a backward model reduction procedure. The procedure involved inclusion of all covariates exhibiting at least a $10 \%$ change in effect on stratified analysis and removal if the absence of the covariate from the model resulted in less than $10 \%$ overall change in the final adjusted measure of effect.

\section{Subanalyses to estimate recall bias}

Recall bias occurs when the dependent variable (cancer worry) or a major confounding variable causes the participants to misclassify the independent variable (FMH solicitation during the past year) in the same direction. This study is susceptible to recall bias; however, simple subanalyses that address the relationship between FMH factors and recall of FMH solicitation can estimate the extent to which such biases are active. Recall is enhanced by the perceived relevance of the exposure to the individual. ${ }^{34}$ Therefore, women with criteria for higher degrees of familial risk (early-onset disease in an FDR, multiple FDRs) would be expected to have more reliable recall than women without such characteristics. If we observe an association between high-risk FH characteristics and recall of FMH solicitation, then we would be more suspicious of recall bias than if we observe no such association. Therefore, we performed crude and adjusted analyses to identify whether the presence of high-risk FH characteristics predicted recall of FMH solicitation within the past year.

\section{Clustering}

We addressed the potential for clustering of both exposure and outcomes by family unit. Our sample contained 353 individuals from 325 families. Individuals within families might share common exposures and attitudes, thus making statistical assumptions of independence faulty. Ignoring such potential clustering primarily results in an underestimation of the true variance of a given variable, but can also influence the point estimate of effect. We performed a bootstrapping procedure ${ }^{35}$ to better estimate the true variance by taking into account fa- 
milial clustering. In this method, we created 500 sets of observations from the original data set, each time sampling only one member from each family. Sampling was done randomly and with replacement. The distribution of coefficients from the analysis of the 500 samples allows an estimate of the variance without the effect of clustering by family.

\section{RESULTS}

\section{Response rate}

Of the 585 women meeting initial eligibility criteria, 476 women returned a completed questionnaire. A total of 353 women met the final eligibility criteria of having and visiting a regular doctor within the past year. We calculated the minimum response rate of $76.4 \%$ as standardized by the American Association for Public Opinion Research. ${ }^{36}$ This assumes that all of the 109 nonresponders would have met final eligibility criteria, and therefore the true response rate is likely to be underestimated. Table 1 compares the study sample with nonresponders. No statistically significant differences in mean age, education, family history, or major ethnicity characteristics were identified.

\section{Descriptive}

Table 1 presents the frequencies and distributions of all study variables. Approximately half of the sample (48\%) reported having FMH solicited during the past year, and approximately one fifth reported no cancer worry over the past 4 weeks.

\section{Main associations}

A report of having an FMH solicited from one's regular doctor during the past year was associated with a lower odds of current cancer worry on both crude (OR 0.53; 95\% CI 0.31, 0.89 ) analyses and analyses adjusted for age, education, higher risk family history characteristics, and clustering by family unit (OR 0.53, 95\% CI 0.30-0.90). We observed an important degree of modification in our estimates of the relationship between FMH solicitation and cancer worry based on whether the woman's family history exhibited higher versus lower risk characteristics. Among women with a family history characterized as high risk, the magnitude of effect was lower and far less precise than among women whose family history did not exhibit any high-risk criteria (Table 2). Among those exposed to FMH solicitation $(\mathrm{N}=163)$, neither mode of FMH collection (i.e., filling our forms vs. direct questioning) was more strongly associated with cancer worry than the other.

\section{Intermediate associations}

Women reported a high perceived severity of developing breast cancer (mean $=4.21 ; \mathrm{SD}=0.95 ; 0-5$ scale) and a somewhat lower perceived likelihood of developing breast cancer someday (mean $=2.75 ; \mathrm{SD}=0.96 ; 0-5$ scale). FMH solicitation within the past year was associated with lower perceived severity of breast cancer (mean difference $=-0.30, P=.01$ ) but not with perceived likelihood of developing breast cancer
Table 1

Description of study sample

\begin{tabular}{|c|c|c|}
\hline Characteristic & $\begin{array}{l}\text { Study Sample } \\
\quad(\mathrm{N}=353)\end{array}$ & $\begin{array}{c}\text { Non-Responders } \\
\quad(\mathrm{N}=109)\end{array}$ \\
\hline & \multicolumn{2}{|c|}{$\mathrm{N}$ (percent) } \\
\hline \multicolumn{3}{|l|}{ Age } \\
\hline mean $(\mathrm{SD})$ & $47.3(10.48)$ & $45.15(13.30)$ \\
\hline \multicolumn{3}{|l|}{ Education } \\
\hline HS grad or less & $48 \quad(10)$ & (13) \\
\hline Some college & $125 \quad(35)$ & $(46)$ \\
\hline College grad & $180 \quad(51)$ & (41) \\
\hline
\end{tabular}

Family history characteristics

Higher risk criteria:

Affected father or brother
FDR with onset $\leq$ age 50 years
Multiple affected FDR's
Any of the higher risk criteria
No higher risk criteria
Non-white
Ashkenazi

FMH solicited within past year

Presence of cancer worry during last 4 weeks

Perceive developing breast cancer to be very serious problem

$\begin{array}{lrl}\text { Strongly agree } & 160 & (46) \\ \text { Agree } & 130 & (37) \\ \text { Somewhat Agree } & 39 & (11) \\ \text { Somewhat Disagree } & 11 & (3) \\ \text { Disagree } & 6 & (2) \\ \text { Strongly Disagree } & 2 & (<1) \\ \begin{array}{l}\text { Perceived likelihood of developing } \\ \text { breast cancer in lifetime }\end{array} & & \\ \text { Certain to happen } & 11 & (3) \\ \text { Very likely } & 56 & (16) \\ \text { Likely } & 144 & (42) \\ \text { Unlikely } & 107 & (31) \\ \text { Very unlikely } & 21 & (6) \\ \text { No chance } & 5 & (1)\end{array}$

SD, standard deviation; FDR, first-degree relative; FMH, family medical history. ${ }^{\star}$ Statistically significant difference at $P<.05$.

(mean difference $=-0.03 ; P=.75)$. No effect modification by family history risk group was demonstrated for either association.

\section{Recall bias analysis}

Women with high-risk family history characteristics were neither more nor less likely to report FMH solicitation than 
Table 2

Crude and adjusted estimates of association between family medical history solicitation and breast cancer worry stratified by family history risk group

\begin{tabular}{lccc}
\hline & Any cancer worry & No cancer worry & OR $(95 \%$ CI $)$ \\
\hline Lower risk family history group $(\mathrm{n}=159)$ & 52 & 27 & $0.44(0.21,0.92)$ \\
FMH solicited & 65 & 15 & -- \\
FMH not solicited & & & $0.47(0.42,0.52)$ \\
Higher risk family history group $(\mathrm{n}=172)$ & 64 & 18 & $0.65(0.30,1.43)$ \\
FMH solicited & 76 & 14 & --
\end{tabular}

FMH, family medical history; OR, odds ratio; CI, confidence interval.Logistic regression model: breast cancer worry $=$ FMH solicited in last year, age, education. Control of clustering within families by repeated random sampling with replacement

those without such FMH characteristics after adjusting for age and education $\left(\mathrm{OR}_{\text {adj }} 0.94 ; 95 \% \mathrm{CI} 0.60-1.45\right)$.

\section{DISCUSSION}

We observed a protective association between recent FMH solicitation in medical practice and the likelihood of currently being bothered by worry about developing breast cancer among women with a close family history of breast cancer. We also found this association to be stronger for women with lower-risk family history characteristics compared with those with high-risk. To our knowledge, this is the first study that describes the potential relationship between screening FMH and cancer worry among women with a close family history of breast cancer.

Little previous work has been done that addresses the psychosocial outcomes of screening family histories for inherited disease risk among undifferentiated patient populations. Qureshi et al. conducted a small randomized trial $(\mathrm{N}=76)$ among a general practice population to assess whether administering an FMH questionnaire (not limited to cancer) resulted in an increase in anxiety. ${ }^{37}$ They observed an increase in shortterm generalized anxiety symptoms and a long-term increase in pessimistic views about one's future health. Leggatt et al. ${ }^{38}$ conducted a descriptive study among 2265 patients of a general practice in England. Individuals were mailed a family history screening questionnaire to identify individuals at high genetic risk of colorectal or breast cancer. Among the 604 patients completing the study, 568 were informed of a screen-negative result. No change from baseline cancer worry was found at 4 weeks postscreening.

Physicians lack confidence in their ability to assess inherited cancer risk. ${ }^{39}$ Our results found no association between FMH solicitation and the perceived likelihood of breast cancer. This suggests that probability feedback subsequent to FMH solicitation might be absent, weak, or generally enough as not to challenge a patient's existing understanding of her risk. ${ }^{40}$ Newly developed pedigree assessment tools will support the physician to provide more explicit probability feedback. Their use in primary care might represent an important change from existing FMH screening processes and will warrant close evaluation of their impact on cancer worry.
In contrast with its impact on perceived likelihood, our findings did identify an association between FMH solicitation and reduced perceptions about the severity of developing breast cancer. Physicians frequently assess FMH in the context of preventive recommendations that emphasize the benefits of detecting cancer early. It is possible that FMH solicitation facilitates a window for optimistic messages about the controllability of breast cancer and therefore alleviates cancer worry. Many emerging FMH screening interventions focus on providing women with feedback about their probability of developing cancer. However, our findings suggest that intervention developers should not underestimate the importance of feedback about the controllability of breast cancer.

This study has important limitations. First, this is a crosssectional study from which causal relationships cannot be clearly understood, and reverse-causality (i.e., "outcome" actually causing "exposure") is of concern. Our observed association is not explained by reverse causality because this would indicate that physicians are preferentially soliciting FMH from women who are not concerned about breast cancer. Our clinical experience suggests that physicians either solicit FMH as part of their usual clinical habits (i.e., true screening test) or as reaction to inquiries, concerns, or symptoms experienced by the patient. Under either of these two scenarios, reverse-causality would be expected to demonstrate a higher level of distress among women whose FMH was solicited.

Second, recall bias is of concern if our dependent variable, worry, affects one's recollection of having her FMH solicited. We performed a subanalysis to estimate what degree of recall bias might be present in this study. Recall is enhanced by the salience of the issue to the individual. ${ }^{34}$ Therefore, if recall bias was a major contributor to our observed associations, we would expect women with higher-risk family histories to recall FMH solicitation to a greater degree than women with lowerrisk family histories. We found no such association on subanalyses and conclude that recall bias is likely not a major threat to validity in this study.

Finally, selection bias is possible. Women registered with the CGN might be more interested in information related to inheritance, be more active in prevention efforts, or demonstrate different levels of cancer worry than other nonregistered women. We sought to minimize such selection bias by recruit- 
ing women only from population-based CGN recruitment centers, not from clinic-based centers that capture women engaged in genetic-related activities. Furthermore, we included only women with a regular source of routine medical care that they have used in the past year. We believe this sample provides a useful first step toward better understanding of the implications that screening FMH has on cancer worry among women with a close family history of breast cancer. More research among less differentiated patient populations is essential to better understand the public health impacts of screening for heritable cancer risk in the routine medical care setting.

\section{ACKNOWLEDGMENTS}

The primary author thanks the CGN Principal Investigators and their research teams for volunteering the substantial resources necessary for completion of this study. The principal author is supported by a grant from the National Cancer Institute (R25 CA87972-02).

\section{References}

1. Yoon PW, Scheuner MT, Peterson-Oehlke KL, Gwinn M, et al. Can family history be used as a tool for public health and preventive medicine? Genet Med 2002;4 (4):304310 .

2. Scheuner MT, Wang SJ, Raffel LJ, Larabell SK, et al. Family history: a comprehensive genetic risk assessment method for the chronic conditions of adulthood. Am J Med Genet 1997;71(3):315-324.

3. Khoury MJ. Genetics and genomics in practice: the continuum from genetic disease to genetic information in health and disease. Genet Med 2003;5(4):261-268.

4. Rich EC, Burke W, Heaton CJ, Haga S, et al. Reconsidering the family history in primary care. J Gen Intern Med 2004;19(3):273-280.

5. Emery J, Rose P. .Expanding the role of the family history in primary care. Br J Gen Pract 1999;49(441):260-261.

6. Acheson LS, Wiesner GL, Zyzanski SJ, Goodwin MA, et al. Family history-taking in community family practice: implications for genetic screening. Genet Med 2000; 2(3):180-185.

7. Carmona RH, Wattendorf DJ. Personalizing prevention: the U.S. Surgeon General's Family History Initiative. Am Fam Physician 2005;71(1):36-39.

8. King MC, Wieand S, Hale K, Lee M, et al. Tamoxifen and breast cancer incidence among women with inherited mutations in BRCA1 and BRCA2: National Surgical Adjuvant Breast and Bowel Project (NSABP-P1) Breast Cancer Prevention Trial. JAMA 2001;286(18):2251-2256.

9. Kauff ND, Satagopan JM, Robson ME, Scheuer L, et al. Risk-reducing salpingooophorectomy in women with a BRCA1 or BRCA2 mutation. N Engl J Med 2002; 346(21):1609-1615.

10. Meijers-Heijboer H, van Geel B, van Putten WL, Henzen-Logmans SC, et al. Breast cancer after prophylactic bilateral mastectomy in women with a BRCA1 or BRCA2 mutation. N Engl J Med 2001;345(3):159-164.

11. Westman J, Hampel H, Bradley T. Efficacy of a touchscreen computer based family cancer history questionnaire and subsequent cancer risk assessment. J Med Genet 2000;37(5):354-360.

12. Emery J, Walton R, Coulson A, Glasspool D, et al. Computer support for recording and interpreting family histories of breast and ovarian cancer in primary care (RAGs): qualitative evaluation with simulated patients. BMJ 1999;319(7201):32-36.

13. Braithwaite D, Sutton S, Smithson WH, Emery J. Internet-based risk assessment and decision support for the management of familial cancer in primary care: a survey of GPs' attitudes and intentions. Fam Pract 2002;19(6):587-590.

14. Coulson AS, Glasspool DW, Fox J, Emery J. RAGs: a novel approach to computerized genetic risk assessment and decision support from pedigrees. Methods Inf Med 2001;40(4):315-322.

15. Family History as a Tool for Public Health and Preventive Medicine. http://www. cdc.gov/genomics/info/conference/famhist.htm. Accessed February 21, 2005.
16. Croyle R. Psychosocial Effects of Screening for Disease Prevention and Detection. New York: Oxford University Press; 1995.

17. Trask PC, Paterson AG, Wang C, Hayasaka S, et al. Cancer-specific worry interference in women attending a breast and ovarian cancer risk evaluation program: impact on emotional distress and health functioning. Psychooncology 2001;10(5): 349-360.

18. Lerman C, Schwartz MD, Lin TH, Hughes C, et al. The influence of psychological distress on use of genetic testing for cancer risk. J Consult Clin Psychol 1997;65(3): $414-420$.

19. Audrain J, Schwartz MD, Lerman C, Hughes C, et al. Psychological distress in women seeking genetic counseling for breast-ovarian cancer risk: the contributions of personality and appraisal. Ann Behav Med 1998;19(4):370-377.

20. Lerman C, Croyle RT. Emotional and behavioral responses to genetic testing for susceptibility to cancer. Oncology (Williston Park) 1996;10(2):191-195, 199; discussion 200-192.

21. Lerman C, Kash K, Stefanek M. Younger women at increased risk for breast cancer: perceived risk, psychological well-being, and surveillance behavior. J Natl Cancer Inst Monogr 1994;(16):171-176.

22. Andersen MR, Smith R, Meischke H, Bowen D, et al. Breast cancer worry and mammography use by women with and without a family history in a populationbased sample. Cancer Epidemiol Biomarkers Prev 2003;12(4):314-320.

23. Burke W, Culver JO, Bowen D, Lowry D, et al. Genetic counseling for women with an intermediate family history of breast cancer. Am J Med Genet 2000;90(5):361368.

24. Nelson N. .Cancer Genetics Network gets under way with 5 years of funding. J Natl Cancer Inst 1997;89(1):10-11.

25. Senior K. New US Cancer Genetics Network announced. Mol Med Today 1998; 4(11):459-460.

26. Anton-Culver H, Ziogas A, Bowen D, et al. The Cancer Genetics Network: recruitment and pilot studies. Community Genet 2003;(6):171-177.

27. Safran DG, Kosinski M, Tarlov AR, Rogers WH, et al. The Primary Care Assessment Survey: tests of data quality and measurement performance. Med Care 1998;36(5): $728-739$.

28. Stange KC, Zyzanski SJ, Smith TF, Kelly R, et al. How valid are medical records and patient questionnaires for physician profiling and health services research? A comparison with direct observation of patients visits. Med Care 1998;36(6):851-867.

29. Lerman C, Daly M, Masny A, Balshem A. Attitudes about genetic testing for breastovarian cancer susceptibility. J Clin Oncol 1994;12(4):843-850.

30. Lerman C, Trock B, Rimer BK, Jepson C, et al. Psychological side effects of breast cancer screening. Health Psychol 1991;10(4):259-267.

31. McHorney CA, Ware JE Jr., Rogers W, Raczek AE, et al. The validity and relative precision of MOS short- and long-form health status scales and Dartmouth COOP charts. Results from the Medical Outcomes Study. Med Care 1992;30(5 Suppl): MS253-MS265.

32. Diefenbach MA, Weinstein ND, O'Reilly J. Scales for assessing perceptions of health hazard susceptibility. Health Educ Res 1993;8(2):181-192.

33. Witte K, Cameron KA, McKeon JK, Berkowitz JM. Predicting risk behaviors: development and validation of a diagnostic scale. J Health Commun 1996;1(4):317-341.

34. Aday LA. Designing and Conducting Health Surveys. Second ed. San Francisco: Jossey-Bass; 1996.

35. Feng Z, McLerran D, Grizzle J. A comparison of statistical methods for clustered data analysis with Gaussian error. Stat Med 1996;15(16):1793-1806.

36. The American Association of Public Opinion Research (AAPOR). Standard Definitions: Final Disposition of Case Codes and Outcome Rates for Surveys. 3rd ed. Lenexa, Kansas; 2004.

37. Qureshi N, Standen PJ, Hapgood R, Hayes J. A randomized controlled trial to assess the psychological impact of a family history screening questionnaire in general practice. Fam Pract 2001;18(1):78-83.

38. Leggatt V, Mackay J, Marteau TM, Yates JR. The psychological impact of a cancer family history questionnaire completed in general practice. J Med Genet 2000;37(6): 470-472.

39. Gramling R, Nash J, Siren K, Eaton C, et al. Family physician self-efficacy with screening for inherited cancer risk. Ann Fam Med 2004;2(2):130-132.

40. Walter FM, Emery J, Braithwaite D, Marteau TM. Lay understanding of familial risk of common chronic diseases: a systematic review and synthesis of qualitative research. Ann Fam Med 2004;2(6):583-594. 